\title{
Regulation of inhomogeneous drilling model with a P-I controller
}

\author{
Alexandre Terrand-Jeanne, Vincent Andrieu, Mélaz Tayakout-Fayolle, Valérie Dos Santos Martins
}

\begin{abstract}
In this paper, we demonstrate that a Proportional Integral controller allows the regulation of the angular velocity of a drill-string despite unknown frictional torque and measuring only the angular velocity at the surface. Our model is an one dimensional damped inhomogeneous wave equation subject to an unknown dynamic at one side while the control and the measurement are in the other side. After writing this system of balance laws into the Riemann coordinates, we design a Lyapunov functional to prove the exponential stability of the closed-loop and show how it implies the regulation of the angular velocity.
\end{abstract}

Index Terms-Lyapunov functional, Regulation, Hyperbolic PDE, Heterogeneous system

\section{INTRODUCTION}

To find and exploit oil, it becomes necessary to dig deeper and deeper below the ground surface. The first consequence of increasing the length of the excavation pipe is the increase of several phenomena causing damage until the breakage of the device. These undesirable phenomena are mainly induced by mechanical oscillations that may appear in axial, radial and lateral directions. According to several studies (i.e [14], [6]), the radial oscillation, namely Stick-slip phenomena, is the most disturbing one. Indeed, it results on angular deformations moving along the pipe, leading to severe damages. It is furthermore the source of other oscillating phenomena (BitBounced and lateral oscillations).

From a mathematical point of view, first studies on this topic were based on lumped parameter models as in [6]. However, increasing the length of tube requires consideration of a distributed parameter model to treat all possible oscillation frequencies.

The control theory for such a mathematical model is still an active research area. Recently, several works exploiting a PDEbased model have been conducted to avoid these oscillation phenomena considering various modelling assumptions and techniques. For example, by using the backstepping approach in [3], [16] ( see also [4] for nonlinear friction terms) or the flatness one in [17]. Another method transforms the whole system into an equivalent time-delay system before ensuring its stability [14]. Note however, that this transformation is impossible when taking into account a distributed damping along the drill pipe.

The main contribution of this article is the analysis of the closed loop stability when the control is provided in the form of a proportional integral (P-I) feedback depending on the topside angular velocity measurement only. This shows that

All authors are with LAGEPP, UMR CNRS 5007, Université Claude Bernard Lyon 1, Université de Lyon, Villeurbanne, France this control regulates the angular velocity of the drill bit to a given reference.

Since the seminal paper of S.A. Pohjolainen in 1982 [15], the problem of output regulation for PDE systems has received a huge interest from the control community. Following this paper, a significant effort has been made to consider a more general class of PDEs and also to relax some crucial assumptions. For instance, it has been shown in [25] that it is possible to relax the compactness requirement on the operator. Moreover, it has been shown in [9] or [26] that it was possible to design a P-I for boundary control for different classes of hyperbolic systems. Following the approach of recent contributions in [23], [24], [19], [2], we prove that regulation and stabilization can be achieved using a Lyapunov approach.

In [3] and [16] the regulation problem of the angular velocity for drilling is also considered. In those works, an observer is built to perform a full state feedback obtained from a backstepping transformation. To compare, our control design is fairly simple since it is a P-I control law which only needs the surface angular velocity measurement. Moreover, our design methodology employs a novel Lyapunov design which should allow nonlinear terms to be taken into account as in [23].

Compare to the preliminary version of the paper which has been presented in [21], we use a more detailed model since we remove one of the assumptions of this previous work : the drilling string is assumed to be inhomogeneous. This requires more involved computations. Moreover, we show how the same control law can handle non dissipative friction terms.

\section{Problem Statement}

\section{A. Regulation of the angular velocity}

In the following, the model that we consider to describe the mechanical oscillation inside the drill pipe of depth $L$ is given as, for $t>0$

$$
\begin{aligned}
& \left.\theta_{t t}(x, t)=\frac{\frac{\partial}{\partial x}\left(G(x) \theta_{x}(x, t)\right)}{\rho}-\beta(x) \theta_{t}(x, t), x \in\right] 0, L[,(1) \\
& G(0) J \theta_{x}(0, t)=c_{a}\left(\theta_{t}(0, t)-\Omega(t)\right) \\
& I_{b} \theta_{t t}(L, t)=-G(L) J \theta_{x}(L, t)-T_{f r}\left(\theta_{t}(L, t)\right)
\end{aligned}
$$

where $\theta:[0, L] \times \mathbb{R}_{+} \rightarrow[0,2 \pi]$ is the angular position of the drill string at point $x$ and time $t$ with respect to a given reference frame. Subscripts $t, x, t t, .$. denote the first or second derivative w.r.t 
variables $t$ or $x$. The mechanical parameters $\operatorname{are}^{1}$ : $G \in C^{1}\left([0, L] ; \mathbb{R}_{+}\right)$: Shear modulus $\quad \rho \in \mathbb{R}_{*}^{+}$: Mass density $\beta \in L^{\infty}\left([0, L] ; \mathbb{R}_{+}\right):$Distributed damping $\quad I_{b} \in \mathbb{R}_{*}^{+}:$BHA inertia $J \in \mathbb{R}_{*}^{+}:$Mass moment of inertia $\quad L \in \mathbb{R}_{*}^{+}:$Pipe's length

$c \in \mathbb{R}_{*}^{+}:$Propagation speed

$c_{a} \in \mathbb{R}_{*}^{+}:$Supplied torque

Finally, $T_{f r}: \mathbb{R} \mapsto \mathbb{R}$ is a function which describes the friction between the drill bit and the earth. Due to the Stribeck effect which occurs at low velocity when the lubrication is not totally effective (see for instance [14]), this function is highly nonlinear for small values of $\left|\theta_{t}(L, t)\right|$. However, when the drill pipe is rotating fast enough, this friction function becomes affine. Hence, in the following we consider that

$$
T_{f r}\left(\theta_{t}(L, t)\right)=c_{b} \theta_{t}(L, t)+T_{0}
$$

where $c_{b}$ is a real number and $T_{0}$ is also an unknown real number which is assumed to be constant. When $c_{b} \geqslant 0$, this implies that the friction dissipates energy. When $c_{b}<0$, the friction terms is a non dissipative terms. In the following both cases are considered.

Compared to the preliminary version of this work it can be noticed that $G$ and $\beta$ are functions of the depth $x$ and are no longer assumed constant all along the string. In fact, the drill pipe is an assemblage of hundreds of pieces of pipe which may have slightly different properties. Furthermore, the temperature and pressure can radically evolve along the pipe and thus modify the thermodynamic properties of the material. Although the drill pipe is considered as a deformable solid, it's mass density and geometry can be supposed constant along the whole pipe. However, according to [10], the Hookes modulus will change with the temperature and pressure variations.

The measured output is the angular velocity of the pipe at the top, that is to say:

$$
y(t)=\theta_{t}(0, t) .
$$

Our control objective is to regulate the velocity at the bottom which is denoted

$$
\underline{y}(t)=\theta_{t}(L, t),
$$

to a given constant reference velocity.

The control problem to be solved is the following : we wish to find a control input $\Omega(t)$ depending only on the measured output such that for every (unknown) constant value of $T_{0}$, the output to regulate $\underline{y}$ is enslaved to a given constant reference value denoted $\underline{y}_{\text {ref }}$.

The structure of the control law is a simple P-I control law. More precisely, the control input is provided by a dynamical error feedback modeled as

$$
\Omega(t)=-k_{p}\left[y(t)-y_{r e f}\right]-k_{i} \eta, \dot{\eta}=y(t)-y_{\text {ref }} \forall t \geq 0 .
$$

In the following, it is shown that given some bounds on the functions $G$ and parameters, $J, \rho, \beta, c_{a}$ and $I_{b}$, there exist $k_{p}$ and $k_{i}$ such that along the solutions of the system (1) with the control input (5), the system is stable and

$$
\lim _{t \rightarrow+\infty}\left|\underline{y}(t)-y_{\text {ref }}\right|=0 \text {. }
$$
$\mathrm{A}$

${ }^{1}$ The modeling assumptions behind these equations are given in Appendix
In a first part of the paper, the model is written in Riemannian coordinates. Then, defining the state space solutions and its topology, we give our main result which shows that regulation is obtained by our P-I control law for a class of hyperbolic PDEs, for which the drilling problem is one illustrative case. The remaining part of the paper is devoted to the demonstration of this result. The proof starts by showing that the regulation property is implied by the exponential stability of the equilibrium state of the closed loop system. To show that the equilibrium state is exponentially stable, we construct a Lyapunov functional.

\section{B. Riemannian coordinates:}

The first step of the study is to project the inhomogeneous drilling model which is given in mechanical coordinates by equations (1)-(4) into normalized Riemannian coordinates. Then the closed loop system is written using the P-I control law (5). In [2], the authors give a general method to reduce linear hyperbolic systems of conservation law with ordinary differential equation (ODE) at their boundaries into a first order transport equation coupled with ODE at their boundaries. Note however, that in eq. (1), $G$ and $\beta$ are functions, hence, we are dealing with systems of balance laws. If the method remains similar, the resulting transport equations are coupled with each other. For $x \in(0,1)$ and $t \geq 0$, let

$$
\begin{aligned}
\phi^{-}(x, t) & =\theta_{t}(L x, L t)-c(x) \theta_{x}(L x, L t), \\
\phi^{+}(x, t) & =\theta_{t}(L x, L t)+c(x) \theta_{x}(L x, L t), \\
z(t) & =\theta_{t}(L, L t), \\
\xi(t) & =\frac{2}{L} \eta(L t),
\end{aligned}
$$

with $c^{2}(x)=\frac{G(L x)}{\rho}$.

The system becomes

$$
\begin{aligned}
& \phi_{t}(x, t)= {\left[\begin{array}{cc}
-c(x) & 0 \\
0 & c(x)
\end{array}\right] \phi_{x}(x, t) } \\
&-\left[\begin{array}{cc}
\lambda(x)+\psi(x) & \lambda(x)-\psi(x) \\
\lambda(x)+\psi(x) & \lambda(x)-\psi(x)
\end{array}\right] \phi(x, t), \\
& \forall x \in(0,1), \\
& \frac{d z}{d t}(t)=-(a+b) z(t)+a \phi^{-}(1, t)+d \\
& \frac{d \xi}{d t}(t)=\phi^{+}(0, t)+\phi^{-}(0, t)-2 y_{\text {ref }}
\end{aligned}
$$

with $\phi(x, t)=\left[\begin{array}{l}\phi^{-}(x, t) \\ \phi^{+}(x, t)\end{array}\right]$. The normalized coefficient are given by

$$
\psi(x)=\frac{d c}{d x}(x)=\frac{\frac{d G}{d x}(L x) L}{2 \sqrt{\rho G(L x)}} \text { and } \lambda(x)=L \frac{\beta(L x)}{2},
$$

and

$$
a=L \frac{G(L) J}{I_{b} c(1)}=\frac{L J}{I_{b}} \sqrt{G(L) \rho}, b=\frac{L c_{b}}{I_{b}}, d=\frac{L T_{0}}{I_{b}}
$$

\footnotetext{
${ }^{2}$ This change of scale in time allows to preserve the diagonal structure with $c(x)$, to normalize the length.
} 
The boundary conditions become

$$
\begin{aligned}
& \phi^{-}(0, t)=\alpha_{0} \phi^{+}(0, t) \\
& \quad+K_{p}\left(\phi^{-}(0, t)+\phi^{+}(0, t)-\tilde{y}_{r e f}\right)+K_{i} \xi(t), \\
& \phi^{+}(1, t)=-\phi^{-}(1, t)+2 z(t),
\end{aligned}
$$

where

$$
\alpha_{0}=\frac{G(0) J-c_{a} c(0)}{G(0) J+c_{a} c(0)},
$$

and the normalized P-I gains $K_{p}, K_{i}$ are given as

$$
K_{p}=\frac{-c_{a} c(0)}{G(0) J+c_{a} c(0)} k_{p} \quad K_{i}=\frac{-L c_{a} c(0)}{G(0) J+c_{a} c(0)} k_{i} .
$$

The normalized reference and the normalized output to be regulated are respectively

$$
\tilde{y}_{r e f}=2 y_{\text {ref }}, \underline{\tilde{y}}(t)=\phi^{-}(1, t)+\phi^{+}(1, t)=2 z(t)
$$

Equations (11)-(13) with boundary conditions (14)-15 define a hyperbolic partial differential equation coupled at the boundaries with two external ODEs.

The state space denoted by $\mathbb{X}$ is the Hilbert space defined as

$$
\mathbb{X}=\left(L^{2}(0,1)\right)^{2} \times \mathbb{R}^{2},
$$

equipped with the norm defined $\forall v=\left(\phi^{-}, \phi^{+}, z, \xi\right)$ in $\mathbb{X}$ as

$$
\|v\|_{\mathbb{X}}=\left\|\phi^{-}\right\|_{L^{2}(0,1)}+\left\|\phi^{+}\right\|_{L^{2}(0,1)}+|z|+|\xi| .
$$

We also introduce a smoother state space

$$
\mathbb{X}_{1}=\left(H^{1}(0,1)\right)^{2} \times \mathbb{R}^{2} .
$$

As has been shown in [2], when $K_{p} \neq 1$, for each initial condition $v_{0}$ in $\mathbb{X}$ which satisfies the boundary conditions (14) and (15), there exists a unique weak solution denoted here by $v$ which belongs to $C^{0}([0,+\infty) ; \mathbb{X})$. Moreover, if the initial condition $v_{0}$ also satisfies the $C^{1}$-compatibility condition (see [2] for more details) and lies in $\mathbb{X}_{1}$ then the solution belongs to the set

$$
C^{0}\left([0,+\infty) ; \mathbb{X}_{1}\right) \cap C^{1}([0,+\infty) ; \mathbb{X})
$$

\section{Main result}

1) Statement of the main result: The main result is separated into two theorems depending on the sign of the friction term $b$.

Theorem 1 (Regulation and stabilization for a dissipative friction term ): Let $0<\underline{a} \leqslant \bar{a}, 0<\bar{b}, 0<\underline{c} \leqslant \bar{c}, 0 \leqslant \bar{\lambda}$, be real numbers. There exist positive real numbers $K_{p} \neq 1, K_{i}$ and $\bar{\psi} \geq 0$ such that for all positive real numbers $a, b$ and for all functions $c, \psi$ and $\lambda$ such that

$$
\begin{gathered}
\underline{a} \leqslant a \leqslant \bar{a}, 0 \leqslant b \leqslant \bar{b} \\
\underline{c} \leqslant c(x) \leqslant \bar{c},|\psi(x)| \leqslant \bar{\psi}, 0 \leqslant \lambda(x) \leqslant \bar{\lambda}, \forall x \in[0,1]
\end{gathered}
$$

for all constant references $\tilde{y}_{r e f}$, all unknowns $d$ and all initial conditions in $\mathbb{X}$, the following holds. (i) It exists an equilibrium state denoted $v_{\infty}$ which is globally exponentially stable in $\mathbb{X}$ for the system (11)-(15). More precisely, there exist $k>0$ and $\nu>0$ such that

$$
\left\|v(t)-v_{\infty}\right\|_{\mathbb{X}} \leqslant k \exp (-\nu t)\left\|v_{0}-v_{\infty}\right\|_{\mathbb{X}}
$$

(ii) If moreover, $v_{0}$ satisfies the $C^{1}$-compatibility conditions and is in $\mathbb{X}_{1}$, the regulation is achieved, i.e.,

$$
\lim _{t \rightarrow+\infty}\left|\underline{\tilde{y}}(t)-\tilde{y}_{r e f}\right|=0 \text {. }
$$

Another theorem can be given when considering set of parameters which allows $b<0$ provided $a+b>0$. Note however that in this case the damping term along the drill pipe (i.e. $\lambda$ ) has to be sufficiently small for our controller to achieve regulation.

Theorem 2 (Regulation and stabilization for unstable friction term): Let $0<\underline{a} \leqslant \bar{a}, \bar{b}>0,0<\underline{\mathfrak{s}}$, be real numbers. There exist positive real numbers $K_{p} \neq 1, K_{i}$ and $\bar{\psi} \geq 0$, $0<\underline{c} \leqslant \bar{c}, 0 \leqslant \bar{\lambda}$, such that for all positive real numbers $a, b$ and for all functions $c, \psi$ and $\lambda$ such that 20 holds and

$$
\underline{a} \leqslant a \leqslant \bar{a}, \underline{\mathfrak{s}}-a \leqslant b \leqslant \bar{b},
$$

for all constant references $\tilde{y}_{r e f}$, all unknowns $d$, the conclusions of Theorem 1 holds.

2) Discussion on the main result: As in [3] or [16], we solve the regulation problem around the equilibrium state by acting on the opposite boundary. An advantage of our approach is that we control the rotatory table and not directly the quantity $\theta_{x}(0, t)$ and that's why only $\theta_{t}(0, t)$ is used to design our controller. Similar to [3], one drawback of our approach compared to [16] is that when non dissipative friction terms are considered (i.e. when $b<0$ ) a strong constraint has to be imposed on the damping term $\lambda(\cdot)$ since it has to be small (in [3] it has to be equal to zero). This constraint on the damping term disappears when considering positive dissipative friction terms.

In addition, the control law obtained is robust with respect to uncertainties on the parameters $a, b, c$ and $\lambda$. Indeed, only the worst case scenario is taken into account to design the control law. In other words, $K_{p}$ and $K_{i}$ depends only on the bounds on those parameters (which can be set as large as desired). This is not the case for the parameter $\psi$ which has to be sufficiently small.

The stability analysis of this kind of models has been considered in [19] or [2]. The dynamics at the top side boundary is due to the integral action of the dynamical control law. The stability analysis of PDE coupled with integral action has been initiated by [15] for parabolic systems (see also [26] for hyperbolic systems) following a spectral analysis. An analysis for $2 \times 2$ hyperbolic PDE has been performed with a Lyapunov approach in [8] and more recently in [22]. Note however that it is not a direct application of this result due to the dynamics at the boundary and that in is a system of balance laws and not a system of conservation laws (see [2]). In the preliminary version of this work [21], we also prove the regulation by a P-I controller but for a simpler model. Here, we take into account functions depending on the 
spatial variable for modeling the distributed damping term and a possible inhomogeneity. The main theorem of our previous study imposed that $\lambda<2$. To conclude, this constraint is now released and one only needs $\lambda$ to be upper bounded and positive. We also demonstrate that even in the case where the wave velocity $c$ is not constant along the pipe, the main theorem holds with a constraint on $\sup _{x \in[0, L]}\left|c_{x}(x)\right|$.

Theorem 1 and 2 establish regulation results for system (11)-(15). Of course these results translate into a regulation result for the drilling system in mechanical coordinates given in (11)-(4). Note however that if the control law ensures asymptotic convergence (in $L^{2}$ or $H^{1}$ ) of $\theta_{t}$ and $\theta_{x}$ toward an equilibrium where the regulation is obtained, nothing is said on the angular position of the drill pipe.

3) A purely integral controller: From a practical point of view, it may not be interesting to use the proportional part of the controller. For instance, and as it has been shown in [1], since $\left|\alpha_{0}\right|<1$, canceling the reflexion with a proportional gain $K_{p}$ may not be an interesting approach due to lack of robustness with respect to input delays. In the following corollary, we show that if $\left|\alpha_{0}\right|<1$ then a purely integral controller solves the problem, provided $\bar{\psi}$ is small enough.

Corollary 1 (Integral controller for stable systems): Assume $\left|\alpha_{0}\right|<1$. Let $0<\underline{a} \leqslant \bar{a}, 0<\bar{b}, 0<\underline{c} \leqslant \bar{c}, 0 \leqslant \bar{\lambda}$, be real numbers. There exist positive real numbers $K_{i}$ and $\bar{\psi} \geq 0$ such that for all positive real numbers $a, b$ and for all functions $c, \psi$ and $\lambda$ such that (19) and 20) hold for all constant references $\tilde{y}_{\text {ref }}$, all unknowns $d$ and all initial conditions in $\mathbb{X}$, Points i), ii) of Theorem 1 hold with $K_{p}=0$.

Proof: The proof of Corollary 1 follows the one of Theorem 1. Only the steps S1, S2 and S3 in the following procedure (proof of the theorem in the following part) for the tuning parameters of the Lyapunov functional are concerned by the restriction $K_{p}=0$.

Therefore, if one selects $\mu$ sufficiently small such that

$$
e^{-2 \mu}>\max \left\{\left(5 \mu \frac{\bar{\lambda}}{\underline{c}}\right)^{2}, \alpha_{0}^{2}\right\}
$$

then $\mu$ respects $\mathrm{S} 1$ ) and it is always possible to find $p$ such that S2) and S3) holds.

It can be noticed that all possible mechanical parameters in system given by equations (1)-(3) leads to parameters $\alpha_{0}$ defined in 16 such that $\alpha_{0}<1$ and $a>0$. Hence, if $c_{b} \leqslant 0$ (in the dissipative friction case) and if we know their extremal values, a purely integral controller can always be employed to solve the regulation problem provided $\psi$ are sufficiently small. Note however that the proportional part (i.e. $K_{p}$ ) allows consideration of larger $\bar{\psi}$.

\section{Proof of Theorems 1 And 2}

To prove the two theorems, we demonstrate that under some conditions, the desired regulation is obtained provided that exists a Lyapunov functional for the system (11)-(15). Then, it only remains to explicitly build the Lyapunov functional to end the proof.

\section{A. Stabilization implies regulation}

In this first subsection, we explicitly give the equilibrium state of the system (11)-(13) with the boundary conditions and (15). We show also that if we assume that $K_{p}$ and $K_{i}$ are selected such that this equilibrium point is exponentially stable along the closed loop, then the regulation is achieved.

1) Definition of the equilibrium: Let $\phi_{\infty}$ be defined as follows.

$$
\begin{aligned}
& \phi_{\infty}^{-}(1)=\frac{a+b}{2 a} \tilde{y}_{r e f}-\frac{d}{a}, \\
& \phi_{\infty}^{+}(1)=y_{\text {ref }}-\phi_{\infty}^{-}(1),
\end{aligned}
$$

and

$$
\phi_{\infty}(x)=\mathcal{R}(x) \phi_{\infty}(1), x \in[0,1]
$$

where $\mathcal{R}$ is the $2 \times 2$ matrix function solution of the ODE

$$
\mathcal{R}_{x}(x)=\frac{1}{c(x)}\left[\begin{array}{cc}
\lambda(x)+\psi(x) & \lambda(x)-\psi(x) \\
-\lambda(x)-\psi(x) & -\lambda(x)+\psi(x)
\end{array}\right] \mathcal{R}(x),
$$

initiated at $x=1$ with $\mathcal{R}(1)=I_{d}$. Note that $\mathcal{R}(x)$ is well defined $\forall x \in[0,1]$ due to the fact that $c(x) \geq \underline{c}>0$ and, $\lambda$ and $\psi$ taking bounded values. We define also

$$
z_{\infty}=\frac{a \phi_{\infty}^{-}(1)+d}{a+b}
$$

and

$$
\xi_{\infty}=\frac{\phi_{\infty}^{-}(0)-\alpha_{0} \phi_{\infty}^{+}(0)}{K_{i}}
$$

It can be checked that $v_{\infty}=\left(\phi_{\infty}^{-}, \phi_{\infty}^{+}, z_{\infty}, \xi_{\infty}\right)$ is an equilibrium for the closed loop system [11)-(13) with the boundary conditions (14) - 15.

2) Sufficient conditions for regulation: In the following, we show that the regulation problem can be rephrased as a stabilization of the equilibrium state.

Proposition 1: Assume that there exist positive real numbers $\omega$ and $L$ and a functional $W: \mathbb{X} \rightarrow \mathbb{R}_{+}$, such that

$$
\frac{\left\|v_{\infty}-v\right\|_{\mathbb{X}}^{2}}{L} \leqslant W(v) \leqslant L\left\|v_{\infty}-v\right\|_{\mathbb{X}}^{2}
$$

Assume moreover that $\forall v_{0} \in \mathbb{X}$ and $\forall t_{0} \in \mathbb{R}_{+}$such that the solution $v$ of (11)-(15) initialized in $v_{0} C^{1}$ at $t=t_{0}$, we have

$$
\dot{W}(v(t)) \leqslant-\omega W(v(t)) .
$$

Then points 1) and 2) of Theorem 1 hold.

Proof: The proof of point 1 ) is by now standard. Let $v_{0}$ be in $\mathbb{X}_{1}$ and satisfying the $C^{0}$ and $C^{1}$-compatibility conditions. It yields that $v$ is smooth for all $t$. Consequently, 27) is satisfied for all $t \geq 0$. With Grönwall lemma, this implies that

$$
W(v(t)) \leqslant e^{-\omega t} W\left(v_{0}\right) .
$$

Hence with (26), this implies that 21) holds with $k=L$ and $\nu=\frac{\omega}{2}$, for any initial conditions in $\mathbb{X}_{1} . \mathbb{X}_{1}$ being dense in $\mathbb{X}$, the result holds also with initial condition in $\mathbb{X}$ and point $i$ ) is satisfied.

Let us show point 2). Note that along solutions of the system (11)-(15), one has

$$
\left(\phi^{-}(x, t)-\phi^{+}(x, t)\right)_{t}=c(x)\left(\phi^{-}(x, t)+\phi^{+}(x, t)\right)_{x} .
$$


Since, at the equilibrium, $\left(\phi^{-}(x, t)-\phi^{+}(x, t)\right)_{t}=0$, and due to the fact that $c(x) \neq 0$ for all $x \in[0,1]$, it yields

$$
\left(\phi_{\infty}^{-}(x)+\phi_{\infty}^{+}(x)\right)_{x}=0 .
$$

This implies that $\phi_{\infty}^{-}(x)+\phi_{\infty}^{+}(x)$ is constant along the string, independently from $x$.

On one hand, with the definition of $\tilde{y}(t)$ in (17) and the definition of the equilibrium, one has

$$
\underline{\tilde{y}}(t)-\tilde{y}_{r e f}=\phi^{-}(1, t)+\phi^{+}(1, t)-\phi_{\infty}^{-}(1)-\phi_{\infty}^{+}(1) .
$$

To show that the equation (22) holds, we need to show that the right hand side of the former equation tends to zero. This may be obtained provided the initial conditions are in $\mathbb{X}_{1}$. Indeed, let $v_{0}$ be in $\mathbb{X}_{1}$ and satisfying $C^{1}$ compatibility conditions. With (18), we know that $v_{t} \in C([0, \infty) ; \mathbb{X})$. Moreover, $v_{t}$ satisfies the dynamics (11)-(15) with $d=0$ and $y_{\text {ref }}=0$ (simply differentiate with time those equations). Hence, $\left\|v_{t}(t)\right\|_{\mathbb{X}}$ converges exponentially toward 0 and in particular

$$
\left\|\phi_{t}^{-}(\cdot, t)\right\|_{L^{2}(0,1)}+\left\|\phi_{t}^{+}(\cdot, t)\right\|_{L^{2}(0,1)} \leq k e^{-\nu t}\left\|\ell_{0}\right\| .
$$

On another hand, denoting $\tilde{\phi}(x, t)=\phi(x, t)-\phi_{\infty}(x)$, employing [11, it yields

$$
\begin{aligned}
& \left\|\phi_{t}^{+}(\cdot, t)\right\|_{L^{2}(0,1)} \geqslant \bar{c}\left\|\tilde{\phi}_{x}^{+}(\cdot, t)\right\|_{L^{2}(0,1)} \\
& -2(\bar{\lambda}+\bar{\psi})\left(\left\|\tilde{\phi}^{-}(\cdot, t)\right\|_{L^{2}(0,1)}+\left\|\tilde{\phi}^{+}(\cdot, t)\right\|_{L^{2}(0,1)}\right),
\end{aligned}
$$

and,

$$
\begin{aligned}
& \left\|\phi_{t}^{-}(\cdot, t)\right\|_{L^{2}(0,1)} \geqslant \bar{c}\left\|\tilde{\phi}_{x}^{-}(\cdot, t)\right\|_{L^{2}(0,1)} \\
& \quad-2(\bar{\lambda}+\bar{\psi})\left(\left\|\tilde{\phi}^{-}(\cdot, t)\right\|_{L^{2}(0,1)}+\left\|\tilde{\phi}^{+}(\cdot, t)\right\|_{L^{2}(0,1)}\right),
\end{aligned}
$$

Consequently $\left\|\tilde{\phi}_{x}^{-}(\cdot, t)\right\|_{L^{2}(0,1)}$ and $\left\|\tilde{\phi}_{x}^{+}(\cdot, t)\right\|_{L^{2}(0,1)}$ also converge to zero. With Sobolev embedding

$$
\sup _{x \in[0,1]}\left|\phi(x, t)-\phi_{\infty}(x)\right| \leqslant C\left\|\phi(\cdot, t)-\phi_{\infty}(\cdot)\right\|_{H^{1}(0,1)},
$$

where $C$ is a positive real number. It implies that

$$
\lim _{t \rightarrow+\infty}\left|\phi^{-}(1, t)+\phi^{+}(1, t)-\phi_{\infty}^{-}(1)-\phi_{\infty}^{+}(1)\right|=0 .
$$

Consequently, with (28), it yields that (22) holds and point ii) is satisfied.

With this proposition in hand and since the system is linear, it turns out that to prove Theorems 1 and 2 it is sufficient to construct a Lyapunov functional. This property does not depend on the value of $y_{\text {ref }}$ and the unknown parameter $d$. So in the following, it is assumed that $y_{r e f}=0, d=0$ and we design a Lyapunov functional.

\section{B. Lyapunov functional construction}

In this subsection a Lyapunov functional is constructed for the system (11)-(15). Due to the complexity of the system considered, the Lyapunov functional is built in three steps. In the first step, a functional $V(\phi(x, t), z(t))$ is introduced and it is shown that it is a Lyapunov functional for (11)-(15) when neglecting the dynamic of $\xi$. In the second step the functional $V(\phi(x, t), z(t))$ is extended to $W(\phi(x, t), z(t), \xi(t))$ and Proposition 5 shows that this extended functional is a Lyapunov function for the system when $\psi(x)=0$. Finally, the function $\psi(x) \neq 0$ is handled with the same Lyapunov functional with a robustness analysis.

1) Step 1: neglecting the $\xi$ dynamic and $\psi(x)=0$ : For this first part, the following PDE system is considered :

$$
\begin{aligned}
\phi_{t}(x, t)= & {\left[\begin{array}{cc}
-c(x) & 0 \\
0 & c(x)
\end{array}\right] \phi_{x}(x, t) } \\
& -\lambda(x)\left[\begin{array}{ll}
1 & 1 \\
1 & 1
\end{array}\right] \phi(x, t), \forall x \in(0,1), \\
\frac{d z}{d t}(t)= & -(a+b) z(t)+a \phi^{-}(1, t),
\end{aligned}
$$

with the boundary conditions

$$
\begin{aligned}
& \phi^{-}(0, t)=\alpha_{p} \phi^{+}(0, t)+\frac{K_{i}}{1-K_{p}} \xi(t), \\
& \phi^{+}(1, t)=-\phi^{-}(1, t)+2 z(t),
\end{aligned}
$$

where, with $K_{p} \neq 1$,

$$
\alpha_{p}=\frac{\alpha_{0}+K_{p}}{1-K_{p}} .
$$

With a slight abuse of notation, we write $V(t)=$ $V(\phi(\cdot, t), z(t))$ and we denote by $\dot{V}(t)$ the derivative of $V$ along the state trajectories (which are $C^{1}$ in time). Inspired by [2], [19], let $V: L^{2}(0,1)^{2} \times \mathbb{R} \mapsto \mathbb{R}_{+}$be the functional defined by:

$$
V(\phi, z)=q z^{2}+\int_{0}^{1} \frac{\phi^{-}(x)^{2}}{c(x)} e^{-\mu x} d x+p \int_{0}^{1} \frac{\phi^{+}(x)^{2}}{c(x)} e^{\mu x} d x .
$$

It is well defined due to the fact that $0<\underline{c} \leqslant c(x)$. In the following it is shown that $K_{p}$ and the parameters of the Lyapunov function $q, p$ and $\mu$ can be selected such that this Lyapunov function satisfies an ISS type inequality. Depending if we are in the context of Theorem 1 or 2 the tuning of the parameters is different and requires two separated Propositions.

Proposition 2: Let $0<\underline{a} \leqslant \bar{a}, 0<\bar{b}, 0<\underline{c} \leqslant \bar{c}, 0 \leqslant \bar{\lambda}$, be real numbers. There exist positive real numbers $K_{p} \neq 1, p$ $\mu, \delta$, and $\omega_{1}$ such that for all parameters $(a, b, c, \lambda)$ satisfying (19) and 20) and $K_{i}$ there exists $q$ such that along the $C^{1}$ solutions of the PDE system (30, 31, 32)

$$
\dot{V}(t) \leqslant-\omega_{1} V(t)+\delta\left|K_{i}\right|^{2}|\xi(t)|^{2}, \forall t \in \mathbb{R}_{+} .
$$

The proof of this proposition is given in Appendix B. The tuning parameters of the Lyapunov functional and $K_{p}$ are selected following this procedure:

S1) $\mu$ is selected sufficiently small such that the inequality (55) is satisfied. Note that in the case in which $\lambda=0, \mu$ can be selected as large as desired and that the larger $\frac{\lambda}{c}$ is, the smaller $\mu$ will be.

S2) $p$ is selected smaller but sufficiently close to $e^{-2 \mu}$ such that inequality $(56)$ is satisfied.

S3) The proportional gain $K_{p}$ is selected sufficiently close to $-\alpha_{0}$ such that employing (33), inequality (44) holds.

S4) $q$ is selected in equation (49). 
S5) Finally, $\omega_{1}$ is selected sufficiently small such that inequalities (51) hold.

In the case in which the friction term $b$ is negative the result is slightly different since only small damping terms $\lambda$ is allowed.

Proposition 3: Let $0<a \leqslant \bar{a}, 0<\overline{\mathfrak{s}}$, be real numbers. There exist positive real numbers $K_{p} \neq 1,0<\underline{c} \leqslant \bar{c}, 0 \leqslant \bar{\lambda}$, $\omega_{1}$ and $\delta$ such that for all parameters $(a, b, c, \lambda)$ satisfying [23) and (20) and $K_{i}$ there exists $q$ such that along the $C^{1}$ solutions of the PDE system (30), (31), 32) inequality (35) is satisfied.

The proof of this proposition is given in Appendix C. The procedure to select the parameter is given as follows.

S'1) $\mu$ and $p$ are selected such the inequality (53) is satisfied (depending on $\overline{\mathfrak{s}}$ and $\underline{a}$.

S'2) $\underline{\lambda}$ and $\bar{c}$ are selected such that inequality 54 holds $\left(\frac{\bar{\lambda}}{\underline{c}}\right.$ has to be small).

S'3) The proportional gain $K_{p}$ is selected sufficiently close to $-\alpha_{0}$ such that employing (33), inequality (44) holds.

S'4) $q$ is selected in equation (49p.

S'5) Finally, $\omega_{1}$ is selected sufficiently small such that inequalities (51) hold.

It can be noticed that when selecting $K_{p}$ in both proposition, it has to ensure that inequality (44) holds. Of course, this implies that inequality 35 holds on a neighborhood $\left[\underline{K}_{p}, \bar{K}_{p}\right]$.

2) Step 2, adding the integral part: In this section, the system (30)-32) to which is also added the dynamics of the integral part of the controller is considered. In other words, the dynamics (13) is considered in the case where $y_{r e f}=0$

$$
\frac{d \xi}{d t}(t)=\phi^{+}(0, t)+\phi^{-}(0, t) .
$$

It is shown in this part of the proof that the integral parameter $K_{i}$ can be tuned based on the construction of a Lyapunov functional. Let : $W: \mathbb{X} \mapsto \mathbb{R}_{+}$be the functional defined by

$$
W(\phi, z, \xi)=V(\phi, z)+r U(\xi, \phi, z)^{2},
$$

where :

$$
U(\xi, \phi, z)=\xi+m^{\top} M(\phi)+n z,
$$

where $r>0, m=\left(m_{1}, m_{2}\right)$ is a vector in $\mathbb{R}^{2}$ and $n$ a real number that will be selected later and $M: L^{1}(0,1) \mapsto \mathbb{R}^{2}$ is an operator defined as

$$
M(\phi)=\int_{0}^{1} \frac{I-R(x)}{c(x)} \phi(x) d x,
$$

with :

$$
R(x)=\int_{0}^{x} \frac{\lambda(s)}{c(s)} d s\left[\begin{array}{ll}
-1 & 1 \\
-1 & 1
\end{array}\right] .
$$

The function $W$ is a Lyapunov functional candidate for the closed loop system since it satisfies the following proposition which proof is given in Appendix $\mathrm{E}$

Proposition 4: There exists $L>0$ such that for all $(\phi, z, \xi)$ in $\mathbb{X}$ we have :

$$
\frac{\|(\phi, z, \xi)\|_{\mathbb{X}}^{2}}{L} \leqslant W(\phi, z, \xi) \leqslant L\|(\phi, z, \xi)\|_{\mathbb{X}}^{2} .
$$

Again, with a slight abuse of notation, we write $W(t)=$ $W(\phi(\cdot, t), z(t), \xi(t))$ and we denote by $\dot{W}(t)$ the time derivative of the Lyapunov function along solutions which are $C^{1}$ in time.

Proposition 5: Assume $\left[\underline{K}_{p}, \bar{K}_{p}\right], \lambda, a, b, \alpha_{0}, p, \mu, q$ and $\omega_{1}$ are given such that equation (35) is satisfied for all $K_{p}$ in $\left[\underline{K}_{p}, \bar{K}_{p}\right]$, then there exist $r, m_{1}, m_{2}, n$ and $K_{i}$ such that if $\psi(x)=0$, then along $C^{1}$ solution of the system given in $(30)$, (31), (32) and (36) the following inequality holds.

$$
\dot{W}(t) \leqslant-\omega_{2} W(t), \forall t \in \mathbb{R}_{+} .
$$

The proof of Proposition 5 is given in Appendix F. In it, the tuning parameters are selected following this procedure:

S6) The parameters $m_{1}, m_{2}$ and $n$ are selected in equation 60 .

S7) $K_{i}$ and $r$ are selected sufficiently small such that 62 , and $(63)$ hold.

Following this route, yields a sufficiently small positive real number $\omega_{2}$.

With Propositions 1, 2, 4 and 5 on one side, and Propositions 1. 34 and 5 on the other side, this yields a proof of Theorem 1 and Theorem 2 in the particular case where $\psi=0$. In the following paragraph, by robustness analysis we show that small $\psi$ can be considered in both theorems.

3) Adding $\psi(x)$ : In this last part of the proof of the theorems, we show that there exists $\bar{\psi}$ such that if $\psi$ is such that $|\psi(x)| \leqslant \bar{\psi}$, the Lyapunov $W$ obtained in Proposition 5 is still decreasing.

Proposition 6: Assume $K_{p}, K_{i}, \lambda, a, b, \alpha_{0}, p, \mu, q$ and $\omega_{2}$ are given such that equation (39) is satisfied along any solutions with $\psi=0$, then there exist $\bar{\psi}$ such that if $|\psi(x)| \leqslant$ $\bar{\psi}$ the following inequality holds

$$
\dot{W}(t) \leqslant-\frac{\omega_{2}}{4} W(t) .
$$

The proof of this proposition which is based on robustness analysis is given in Appendix G To summarize, with Propositions 1, 2 4, 5 and 6, the proof of Theorem 1 is completed. Employing Proposition 3 instead of Proposition 5 proves Theorem 2

The selection of $\bar{\psi}$ is the last part of the algorithm.

S8) The computation of $\bar{\psi}$ is given in 65].

\section{DISCUSSION ON THE RESULTS}

A. Numerical selection of $K_{p}$ and $K_{i}$ with the same Lyapunov functional

We test the procedure for several values of $\mu, p$ and $K_{p}$ respecting S1) to S3) in the case of Proposition 2 with a dissipative friction term (i.e. when $b \geqslant 0$ ).

The greatest gain found for $K_{i}$ is $K_{i}^{*}=1,13$ with $K_{p}=$ $0.47\left(\alpha_{p}=-0.5\right), \mu=0.0375, q=10^{-3}, w_{1}=0.099$, $p=0.76$. However this value of $K_{i}^{*}$ leads to

$$
k_{i}=5.7437 \times 10^{-4}
$$

From this maximal value of $K_{i}^{*}$, it is possible to select a smaller $K_{i}$ such that one can include $\psi(x)$ in the stability analysis. The resulting $\bar{\psi}(x)$ is then determined using $\mathbf{S} 8$ ). 
It is worth noting that these gains are far from the optimal value which is expected since Lyapunov analysis is a very conservative approach in control theory.

B. Comparative study with time delay system tools $(\lambda(x)=$ $0, c(x)=c, b \geqslant 0$ )

Selecting $\lambda(x)=0$ and $c(x)=c$ constant, the system (11)-(15) become a pure system of two linear conservation law coupled with linear ODEs at boundaries. In this particular case, the stability analysis can be performed employing timedelay tools. Backstepping approach has also been considered in [5] allowing non dissipative friction terms. In the following, to evaluate the conservatism of our Lyapunov approach, we restrict our attention to the stability property and forget about the regulation. Hence, we consider the case in which $y_{\text {ref }}=0$ and $d=0$.

Proposition 7: If $\lambda(x)=0, c(x)=c$ constant, $b \geqslant 0$ and provided $K_{p} \in\left(-\infty ; \frac{1-\alpha_{0}}{2}\right)$ and $K_{i}\left(K_{p}-1\right)>0$, there exist $k>0$ and $\nu>0$ such that for all initial condition solutions of system (11)-(15) satisfy :

$$
\|v(t)\|_{L^{\infty}[0,1]} \leqslant k \exp (-\nu t)\left\|v_{0}\right\|_{L^{\infty}[0,1]} ;
$$

The proof is obtained following these three steps.

1) Show that the poles of the system [11)-(15) $\lambda(x)=0$, $c(x)=c$ satisfy the following characteristic equation

$$
(s+a+b)(s+K)+(s+b-a)\left(s \alpha_{p}-K\right) e^{-2 c s}=0,
$$

where $K=\frac{-K_{i}}{1-K_{p}}$

2) Apply the Walton and Marshall procedure as in [2, Sec 3.4.3] to conclude on the pole stability;

3) The equivalence between exponential stability of the $L^{\infty}$-norm and the stability of the poles is given by [2, Theorem 3.14].

Computation of the two first step are given in Appendix $\mathrm{H}$ while reader is referred to [18] for more details on the Walton and Marshall procedure.

It is interesting to remark that the Lyapunov approach is very conservative compared to the result we can be get following a frequency approach since no bounds on the integral term is imposed. An interesting study would be to follow the tools introduced in [7] to reduce the conservatism of the Lyapunov approach.

\section{Simulation}

The numerical scheme used is a semi-discretization in space of the equation (1) with 101 space nodes. The boundary conditions at $x=0$ and $x=L$ are numerically taken into account by using a ghost node method (see in [11] for more details about this method). Values of the parameters are chosen following 12 and reported in Table I

The friction $\beta(x)$ models both possible rubbing between the drill pipe and the drill holes and constant dynamic friction. The function $G(x)$ is supposed to be equal to $G_{0}$ at $x=0$ and decreases along the drill pipe as the temperature and pressure increase. Figure 1 depicts the shape of those functions. In the Figures 2 and 3 , we compare the regulation action for different

\begin{tabular}{|c|r||c|r|}
\hline Name & Values & Name & Values \\
\hline$G$ & $79.6 \times 10^{9}{\mathrm{~N} . \mathrm{m}^{-2}}^{-2}$ & $\rho$ & $7850 \mathrm{~kg} . \mathrm{m}^{-3}$ \\
$J$ & $1.19 \times 10^{-5} \mathrm{~m}^{4}$ & $\beta$ & $0.05 \mathrm{~kg} . \mathrm{m} . \mathrm{s}^{-1}$ \\
$c_{a}$ & $2000 \mathrm{~N} . \mathrm{m} . \mathrm{s.rad} \mathrm{rad}^{-1}$ & $L$ & $2000 \mathrm{~m}$ \\
$I_{b}$ & $311 \mathrm{~kg} . \mathrm{m}^{-2}$ & $c$ & $3184.3 \mathrm{m.s}$ \\
$c_{b}$ & $0.03 \mathrm{~kg} . \mathrm{s}^{-1}$ & $T_{0}$ & $7500 \mathrm{~N}$ \\
\hline
\end{tabular}

TABLE I

VALUES OF THE PHYSICAL COEFFICIENTS

values of $k_{i}$ when considering an affine law for the friction acting at $x=L$, i.e with $T_{f r}\left(\theta_{t}(L, t)\right)=c_{b} \theta_{t}(L, t)+T_{0}$. On Figure 2 we do not use proportional action $\left(k_{p}=0\right)$ while in Figure 3 we select $k_{p}=-0.5$. In the figure 4, we simulate the system for $k_{i}=k_{p}=0$ in order to compare the regulation with the uncontrolled case, still considering an affine friction law.

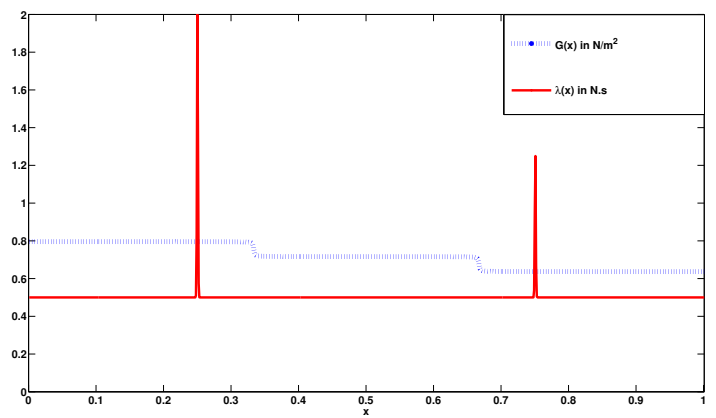

Fig. 1. Shape of the function $\lambda(x)$ and $G(x)$

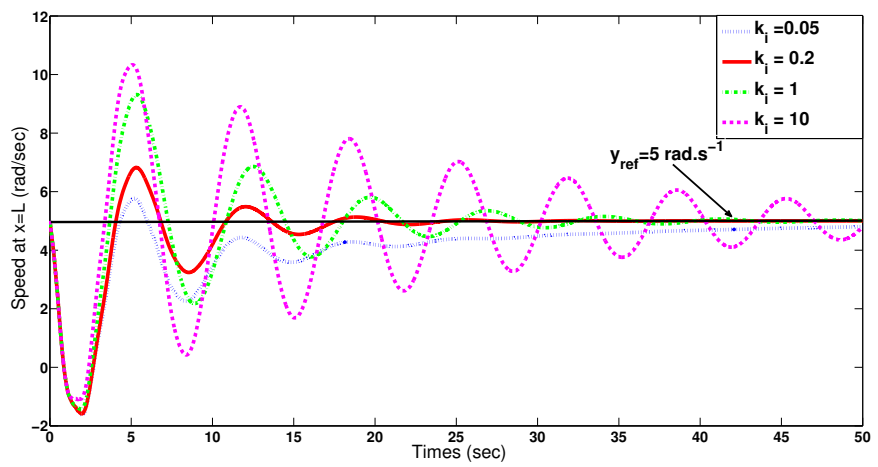

Fig. 2. Pure integral controller with $k_{i}=0.05,0.2,1,10$

It is worth pointing out that in some cases, the proportional action help to stabilize the Stick-Slip phenomenon occurring in drilling system. Figure 5 depicts the nonlinear model in closed-loop with four different proportional gain $k_{p}$ and same integral gain $k_{i}=0.2$ starting from the same initial condition corresponding to stick-slip phenomena. In those cases, we use the following non-linear friction law (see [14])

$$
\begin{aligned}
& T_{f r}\left(\theta_{t}(L, t)\right)=c_{b} \theta_{t}(L, t) \\
& \quad+\frac{2 T_{0}}{\pi}\left(\alpha_{1} \theta_{t}(L, t) e^{\left|\alpha_{2}\right| \theta_{t}(L, t)}+\arctan \left(\alpha_{3} \theta_{t}(L, t)\right)\right.
\end{aligned}
$$




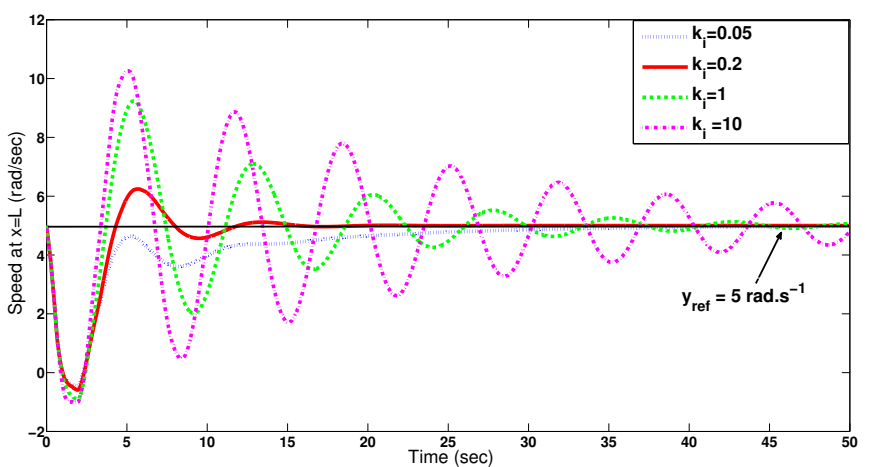

Fig. 3. P-I controller with $k_{p}=-0.5$ and $k_{i}=0.05,0.2,1,10$

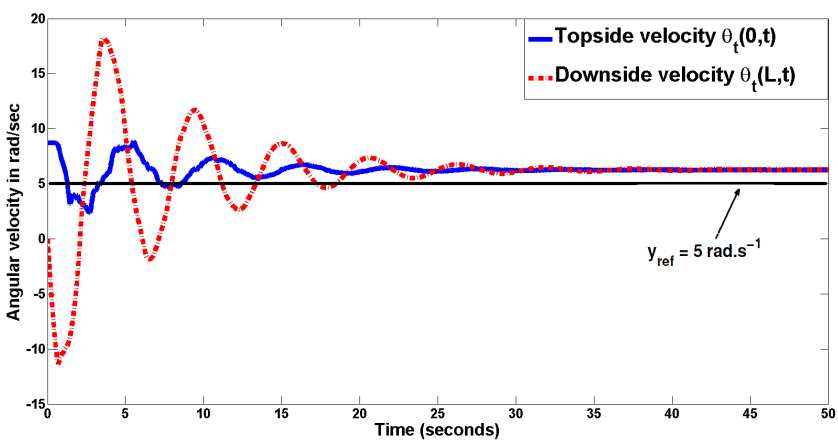

Fig. 4. Evolution of the boundary angular velocities without controller

In some case regulation is still effective, whereas, in the case of a pure integral controller, regulation is not achieved due to the non-linearity and so the mechanical oscillations go on.

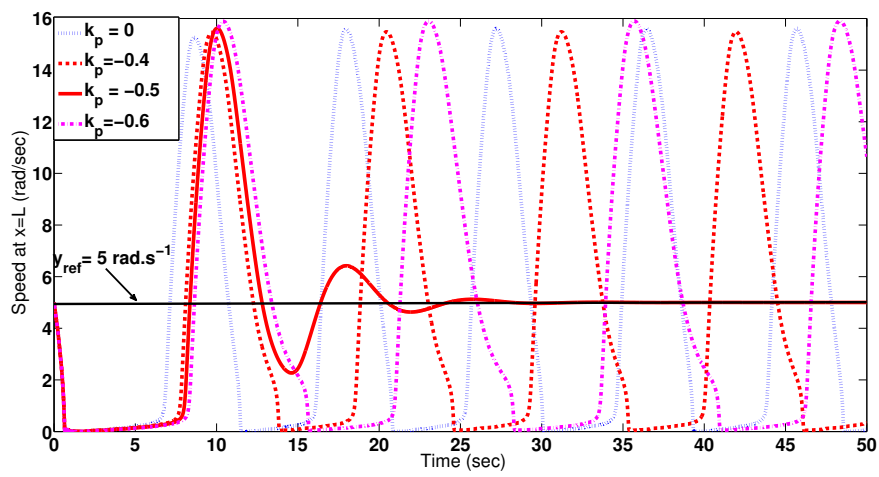

Fig. 5. Non-linear model with P-I controller $k_{p}=[0 ;-0.4 ;-0.5 ;-0.6]$ and $k_{i}=0.2$

\section{CONCLUSION}

This paper presents an analysis of a P-I controller to regulate the bottom velocity of a drill pipe. The model used for describing the dynamics of the drill pipe is extended to the case of a damped wave equation (as in [16], [14]). It has been shown that exponential stability of the equilibrium and output regulation could be achieved with this kind of control law even if the considered model is a coupled inhomogeneous PDE model. The result has been obtained employing a novel construction of Lyapunov functional, inspired from forwarding methods used for the control of nonlinear system, which is valid for all admissible mechanical parameters. Comparing with the case of a system of conservation laws (see Proposition 77, it is clear that the Lyapunov design imposes a small value of the integral gain $K_{i}^{*}$ which can be seen as very restrictive. However, an example using real data experiment (see fig 2 ) tends to show that $k_{i} \approx 0.2$ seems to be the best choice for the rate convergence of the variable to be regulated.

An interesting topic for future work would be to address the regulation of a drilling model with time varying references.

\section{REFERENCES}

[1] J. Auriol, U. J. F. Aarsnes, P. Martin, and F. Di Meglio. Delayrobust control design for heterodirectional linear coupled hyperbolic pdes. IEEE Transactions on Automatic Control, pages 1-1, 2018.

[2] G. Bastin and J.-M. Coron. Stability and boundary stabilization of 1-d hyperbolic systems, volume 88. Springer, 2016.

[3] H.I Basturk. Observer-based boundary control design for the suppression of stick-slip oscillations in drilling systems with only surface measurements. Journal of Dynamic Systems, Measurement, and Control, 139(10):104501, 2017.

[4] Nikolaos Bekiaris-Liberis and Miroslav Krstic. Compensation of wave actuator dynamics for nonlinear systems. IEEE Transactions on Automatic Control, 59(6):1555-1570, 2014.

[5] Delphine Bresch-Pietri and Miroslav Krstic. Adaptive output-feedback for wave pde with anti-damping-application to surface-based control of oil drilling stick-slip instability. In Decision and Control (CDC), 2014 IEEE 53rd Annual Conference on, pages 1295-1300. IEEE, 2014.

[6] C. Canudas-de Wit, F. R Rubio, and M. A. Corchero. D-oskil: A new mechanism for controlling stick-slip oscillations in oil well drillstrings. IEEE Transactions on Control Systems Technology, 16(6):1177-1191, 2008.

[7] Jean-Michel Coron and Amaury Hayat. PI controllers for 1-D nonlinear transport equation. working paper or preprint, April 2018

[8] V. Dos Santos, G. Bastin, J.-M. Coron, and B. d'Andrea Novel. Boundary control with integral action for hyperbolic systems of conservation laws: Lyapunov stability analysis and experimental validation. Automatica, 44(5)(1):1310 - 1318, 2008.

[9] V. Dos Santos, Y. Touré, E. Mendes, and E. Courtial. Multivariable boundary control approach by internal model, applied to irrigation canals regulation. 16th IFAC World Congress, IFAC Proceedings Volumes, 38(1):63-68, 2005.

[10] M. W. Guinan and D. J. Steinberg. Pressure and temperature derivatives of the isotropic polycristalline shear modulus for 65 elements. Physics and Chemical Solids, 35:1501-1512, 1974.

[11] M.H. Holmes. Introduction to numerical methods in differential equations. Texts in applied mathematics. Springer, 2006.

[12] JD Jansen and L Van den Steen. Active damping of self-excited torsional vibrations in oil well drillstrings. Journal of sound and vibration, 179(4):647-668, 1995.

[13] J. Lubliner and P. Papadopoulos. Introduction to Solid Mechanics : An Integrated Approach. Springer, 2014.

[14] M. B. S. Márquez, I. Boussaada, H. Mounier, and S.-I. Niculescu. Analysis and Control of Oilwell Drilling Vibrations: A Time-Delay Systems Approach. Springer, 2015.

[15] S. Pohjolainen. Robust multivariable pi-controller for infinite dimensional systems. IEEE Transactions on Automatic Control, 27(1):17-30, 1982.

[16] C. Roman, D. Bresch-Pietri, E. Cerpa, C. Prieur, and O. Sename. Backstepping observer based-control for an anti-damped boundary wave pde in presence of in-domain viscous damping. In Decision and Control (CDC), 2016 IEEE 55th Conference on, pages 549-554, Las Vegas, USA, 2016. IEEE.

[17] C. Sagert, F. Di Meglio, M. Krstic, and P. Rouchon. Backstepping and flatness approaches for stabilization of the stick-slip phenomenon for drilling. IFAC Proceedings Volumes, 46(2):779 - 784, 2013. 5th IFAC Symposium on System Structure and Control.

[18] G. J Silva, A. Datta, and S. P Bhattacharrya. PID controllers for timedelay systems. Control Engineering. Birkhäuser Boston, 2004.

[19] Y. Tang and G. Mazanti. Stability analysis of coupled linear odehyperbolic pde systems with two time scales. Automatica, 85(Supplement C):386 - 396, 2017. 
[20] A. Terrand-Jeanne and V. Dos Santos Martins. Modelings approaches for stick-slip phenomena in drilling. volume 49, pages 118-123, Bertinoro, Italy, 2016. Elsevier.

[21] A. Terrand-Jeanne, V. Dos Santos Martins, and V. Andrieu. Regulation of the downside angular velocity of a drilling string with a p-i controller. In Proceedings of European Control Conference, Limassol, Cyprus, 2018.

[22] N.-T. Trinh, V. Andrieu, and C.-Z. Xu. Multivariable pi controller design for $2 \times 2$ systems governed by hyperbolic partial differential equations with lyapunov techniques. In Decision and Control (CDC), 2016 IEEE 55th Conference on, pages 5654-5659, Las Vegas, USA, 2016. IEEE.

[23] N.-T. Trinh, V. Andrieu, and C.-Z. Xu. Design of integral controllers for nonlinear systems governed by scalar hyperbolic partial differential equations. IEEE Transactions on Automatic Control, 2017.

[24] Ngoc-Tu Trinh, Vincent Andrieu, and Cheng-Zhong Xu. Output regulation for a cascaded network of $2 \times 2$ hyperbolic systems with pi controller. Automatica, 91:270-278, 2018.

[25] C.-Z. Xu and H. Jerbi. A robust pi-controller for infinite-dimensional systems. International Journal of Control, 61(1):33-45, 1995.

[26] C.-Z. Xu and G. Sallet. Multivariable boundary pi control and regulation of a fluid flow system. Mathematical Control and Related Fields, 4(4):501-520, 2014.

\section{APPENDIX}

\section{A. Modeling}

In this subsection some explanation is given on the way equation (1) is obtained to model the drill pipe dynamics. There exist several approaches to model the behavior of mechanical deformations along the drill pipe in order to synthesize a control policy. Early studies have considered lumped parameter models of two or more states (see [12], [6]). If these models allow a wide choice of control strategies, they cannot take into account all possible vibration modes (see [20]). However, it is well known (see for instance [13, Chap 7]) that when studying an infinitesimal slice of pipe with constant geometry and properties, it is possible to express the torque (in N.m) induced by an angular deformation as

$$
T(x, t)=G J \theta_{x}(x, t) .
$$

The considered model has to take into account space varying mechanical parameters and an external damping force acting along the drill pipe. Consider an infinitesimal slice of pipe between coordinates $x$ and $x+d x$. The mechanical power balance into this slice yields

$$
\frac{d}{d t} E_{c}(x, t)=P_{i n t}+P_{e x t}
$$

Meaning that the variation of kinetic energy correspond to the sum of intern and extern power. If one supposes that the Hookes modulus may depends on the depth (and so on the temperature and pressure along the drill hole [10]), the internal power (in Watts) due to traveling torsion is written

$$
\begin{aligned}
P_{\text {int }} & =P_{\text {in }}-P_{\text {out }}=(T(x, t)-T(x+d x, t)) \theta_{t}(x, t) \\
& =\left(G(x) \theta_{x}(x, t)-G(x+d x) \theta_{x}(x+d x, t)\right) J \theta_{t}(x, t)
\end{aligned}
$$

The distributed damping implies :

$$
P_{\text {ext }}=-2 \pi r \sigma(x) \theta_{t}^{2}(x, t) d x \text { in Watts }
$$

where $\sigma(x)$ in N.s. $m^{-1}$ refers to a surface friction coefficient, and the kinetic energy of this slice of pipe

$$
E_{c}(x, t)=\rho J \theta_{t}^{2}(x, t) d x \text { in Joules }
$$

So, considering the approximation

$\frac{G(x) \theta_{x}(x, .)-G(x+d x) \theta_{x}(x+d x, .)}{d x} \approx \partial_{x}\left(G(x) J \theta_{x}(x,).\right)$

it yields that

$$
\theta_{t t}(x, t)=\frac{\frac{\partial}{\partial x}\left(G(x) \theta_{x}(x, t)\right)}{\rho}-\beta(x) \theta_{t}(x, t)
$$

with $\beta(x)=\frac{2 \pi r \sigma(x)}{\rho J}$.

Variables employed are summarized in Table I

\section{B. Proof of Proposition 2}

The time derivative of $V$ satisfies

$$
\begin{aligned}
\dot{V}(t)=-w_{0}(t)^{T} \mathcal{P} w_{0}(t)-w_{1}(t)^{T} \mathcal{M} w_{1}(t) & \\
& -\int_{0}^{1} \phi(x, t)^{T} \mathcal{N} \phi(x, t) d x
\end{aligned}
$$

where

$$
w_{0}(t)=\left(\phi^{+}(0, t) \quad K_{i} \xi(t)\right)^{T}, w_{1}(t)=\left(\phi^{-}(1, t) \quad z(t)\right)^{T},
$$

and :

$$
\begin{aligned}
\mathcal{M} & =\left[\begin{array}{cc}
e^{-\mu}-p e^{\mu} & 2 p e^{\mu}-a q \\
2 p e^{\mu}-a q & 2(a+b) q-4 p e^{\mu}
\end{array}\right], \\
\mathcal{N} & =\left[\begin{array}{cc}
\left(2 \frac{\lambda(x)}{c(x)}+\mu\right) e^{-\mu x} & \frac{\lambda(x)}{c(x)}\left(e^{-\mu x}+p e^{\mu x}\right) \\
\frac{\lambda(x)}{c(x)}\left(e^{-\mu x}+p e^{\mu x}\right) & p\left(2 \frac{\lambda(x)}{c(x)}+\mu\right) e^{\mu x}
\end{array}\right], \\
\mathcal{P} & =\left[\begin{array}{cc}
p-\alpha_{p}^{2} & -\frac{\alpha_{p}}{\left(1-K_{p}\right)} \\
-\frac{\alpha_{p}}{\left(1-K_{p}\right)} & -\frac{1}{\left(1-K_{p}\right)^{2}}
\end{array}\right],
\end{aligned}
$$

In the proof, the matrices $\mathcal{M}, \mathcal{N}$ and $\mathcal{P}$ are considered separately.

The matrix $\mathcal{N}$ : Note that

$$
\begin{aligned}
& \operatorname{det}(\mathcal{N}(x))=p\left(2 \frac{\lambda(x)}{c(x)}+\mu\right)^{2}-\left(\frac{\lambda(x)}{c(x)}\left(e^{-\mu x}+p e^{\mu x}\right)\right)^{2} \\
& =F(p, \mu, x)\left[\sqrt{p}\left(2 \frac{\lambda(x)}{c(x)}+\mu\right)+\frac{\lambda(x)}{c(x)}\left(e^{-\mu x}+p e^{\mu x}\right)\right] .
\end{aligned}
$$

where

$$
F(p, \mu, x)=\sqrt{p}\left(2 \frac{\lambda(x)}{c(x)}+\mu\right)-\frac{\lambda(x)}{c(x)}\left(e^{-\mu x}+p e^{\mu x}\right) .
$$

Hence, with Lemma 1 in Appendix D there exist $p$ and $\mu$ (depending on $\bar{\lambda}$ and $\underline{c}$ ) such that

$$
0<p<e^{-2 \mu}
$$

and $F(p, \mu, x)>0$. So, $\mathcal{N}>0$.

The matrix $\mathcal{P}$ : We pick $K_{p}$ in 33 such that

$$
p-\alpha_{p}^{2}>0 \text {. }
$$

Then there exists a positive real number $\delta$ such that

$$
-w_{0}(t)^{T} \mathcal{P} w_{0}(t) \leqslant \delta\left|K_{i} \xi(t)\right|^{2} .
$$

The matrix $\mathcal{M}$ : With 43, $\mathcal{M}>0$ if and only if

$$
f(q)>0
$$


where :

$$
\begin{aligned}
f(q) & =\left(e^{-\mu}-p e^{\mu}\right) q\left(2(a+b)-4 p e^{\mu}\right)-\left(2 p e^{\mu}-a q\right)^{2} \\
& =-a^{\prime} q^{2}+b^{\prime} q-c^{\prime},
\end{aligned}
$$

and $a^{\prime}, b^{\prime}$ and $c^{\prime}$ are positives real numbers given as :

$$
\begin{aligned}
& a^{\prime}=a^{2} \\
& b^{\prime}=\left(e^{-\mu}-p e^{\mu}\right) 2(a+b)+4 p a e^{\mu} \\
& c^{\prime}=\left(e^{-\mu}-p e^{\mu}\right) 4 p e^{\mu}+4 p^{2} e^{2 \mu}=4 p
\end{aligned}
$$

This function $f(q)$ is a second order polynomial, whose maximum is reached for $q=\frac{b^{\prime}}{2 a^{\prime}}$. Note that, $f\left(\frac{2 b^{\prime}}{2 a^{\prime}}\right)$ is strictly positive if and only if

$$
b^{\prime 2}-4 a^{\prime} c^{\prime}=\left(b^{\prime}-2 \sqrt{a^{\prime} c^{\prime}}\right)\left(b^{\prime}+2 \sqrt{a^{\prime} c^{\prime}}\right)>0 .
$$

Since $a^{\prime}, b^{\prime}$ and $c^{\prime}$ are positives, it remains to verify that $b^{\prime}-$ $2 \sqrt{a^{\prime} c^{\prime}}$ is positive. Keeping in mind that with 43$),\left(e^{-\mu}-\right.$ $\left.p e^{\mu}\right)>0$, it yields

$$
\begin{aligned}
\frac{b^{\prime}-2 \sqrt{a^{\prime} c^{\prime}}}{\left(e^{-\mu}-p e^{\mu}\right)} & =2(a+b)-\frac{4 a\left(\sqrt{p}-p e^{\mu}\right)}{e^{-\mu}-p e^{\mu}} \\
& =2 a\left(1-\frac{2 \sqrt{p}}{e^{-\mu}+\sqrt{p}}\right)+2 b .
\end{aligned}
$$

Since $\frac{2 \sqrt{p}}{e^{-\mu}+\sqrt{p}}<1$, it yields $f\left(\frac{2 b^{\prime}}{2 a^{\prime}}\right)>0$, consequently, we set as a function of $(a, b)$ as

$$
q=\frac{2 b^{\prime}}{2 a^{\prime}}=\frac{\left(e^{-\mu}-p e^{\mu}\right) 2(a+b)+4 p a e^{\mu}}{2 a^{2}},
$$

and $\mathcal{M}>0$.

Conclusion: Consequently,

$$
\mathcal{M}>0, \mathcal{N}(x)>0, \forall x \in[0,1],
$$

then, employing the fact that $\mathcal{N}$ is continuous, $q$ is a continuous function of $a$ and $b$ (which belong to a compact set) and $c$ is upper bounded, there exists $\omega_{1}$ in $\mathbb{R}_{+}$(depending on $\underline{a}$, $\bar{a}$, and $\underline{b}$ ) such that for all $a, b$ these matrix inequalities

$$
\mathcal{M} \geqslant \omega_{1} q I, \mathcal{N}(x) \geqslant \frac{\omega_{1}}{c(x)}\left[\begin{array}{ll}
1 & 0 \\
0 & p
\end{array}\right],
$$

are satisfied. This implies that 35 holds.

\section{Proof of Proposition 3}

The proof follows mainly the same lines as the one of Proposition 2. The only difference comes from the analysis of the matrices $\mathcal{M}$ and $\mathcal{P}$.

The matrix $\mathcal{M}$ : Following the proof of Proposition 2 we consider the case in which (43) holds. In that case, inequality (47) satisfies

$$
\frac{b^{\prime}-2 \sqrt{a^{\prime} c^{\prime}}}{\left(e^{-\mu}-p e^{\mu}\right)}=2(a+b)-4 a \frac{\sqrt{p}}{e^{-\mu}+\sqrt{p}} .
$$

Picking $p$ and $\mu$ such that

$$
\sqrt{p} \frac{2 \bar{a}-\underline{\mathfrak{s}}}{\underline{\mathfrak{s}}}<e^{-\mu},
$$

yields $\sqrt{p} \frac{a-b}{a+b}<e^{-\mu}$ for all $(a, b)$ satisfying 23. Consequently $\frac{2 \sqrt{p}}{e^{-\mu}+\sqrt{p}}<\frac{a+b}{a}$ and $b^{\prime}-2 \sqrt{a^{\prime} c^{\prime}}>0$ for all $(a, b)$ satisfying (23). It yields with $q$ defined in (49) that $\mathcal{M}>0$. The matrix $\mathcal{N}$ : Note that the function $F$ defined in 42 satisfies for all $x$ in $[0,1]$ :

$$
F(p, \mu, x) \geqslant 2 \sqrt{p} \mu-\frac{\bar{\lambda}}{\underline{c}}\left(2 \sqrt{p}+1+p e^{\mu}\right) .
$$

Hence, selecting $\frac{\bar{\lambda}}{\underline{c}}$ such that

$$
\frac{\bar{\lambda}}{\underline{c}}<\frac{2 \sqrt{p} \mu}{2 \sqrt{p}+1+p e^{\mu}},
$$

yields $\mathcal{N}(x)>0$ for all $x$ in $[0,1]$ and functions $\lambda$ and $c$ satisfying (20). The rest of the proof follows the one of Proposition 2 .

\section{Technical Lemmas}

Lemma 1: Consider the mapping $(p, \mu, x) \in \mathbb{R}_{+} \times \mathbb{R}_{+} \times$ $[0,1] \rightarrow \mathbb{R}$ given by

$$
F(p, \mu, x)=\sqrt{p}\left(2 \frac{\lambda(x)}{c(x)}+\mu\right)-\frac{\lambda(x)}{c(x)}\left(e^{-\mu x}+p e^{\mu x}\right),
$$

where

$$
0 \leqslant \lambda(x) \leqslant \bar{\lambda}, 0<\underline{c} \leqslant c(x) .
$$

Then, for all $\mu$ such that

$$
\mu>0, \frac{e^{-\mu}}{\mu}>5 \frac{\bar{\lambda}}{\underline{c}},
$$

there exists $p$ such that

$$
e^{-2 \mu}>p>\max \left\{C_{1}(\mu), C_{2}(\mu), C_{3}(\mu)\right\},
$$

where $C_{i}, i=1,2,3$ are given as

$$
\begin{aligned}
& C_{1}(\mu)=\left(\frac{4}{5}\right)^{2} e^{-2 \mu}, \\
& C_{2}(\mu)=\max \left\{e^{-\mu}-\frac{c}{\bar{\lambda}} \frac{e^{-\mu}}{10} \mu, 0\right\}^{2}, \\
& C_{3}(\mu)=e^{-2 \mu} \max \left\{1-\frac{\underline{c}}{5 \bar{\lambda}} \mu, 0\right\} .
\end{aligned}
$$

Moreover, for such couple $(\mu, p)$ and for all $x$ in $[0,1]$

$$
F(p, \mu, x)>\frac{e^{-\mu} \mu}{5} .
$$

Proof: Consider the function $G: \mathbb{R}_{+} \times[0,1] \rightarrow \mathbb{R}$ defined as

$$
\begin{aligned}
G(\mu, x) & =e^{\mu} F\left(e^{-2 \mu}, \mu, x\right)-\mu \\
& =2 \frac{\lambda(x)}{c(x)}-\frac{\lambda(x)}{c(x)}\left(e^{\mu(1-x)}+e^{\mu(x-1)}\right) \\
& =\frac{\lambda(x)}{c(x)}\left(2-e^{\mu(1-x)}-e^{\mu(x-1)}\right) .
\end{aligned}
$$

Note that $G(0, x)=0$ and moreover

$$
G_{\mu}(\mu, x)=\frac{\lambda(x)}{c(x)}\left(-(1-x) e^{\mu(1-x)}-(x-1) e^{\mu(x-1)}\right)
$$

which gives $G_{\mu}(0, x)=0$. Also,

$$
G_{\mu \mu}(\mu, x)=-(1-x)^{2} \frac{\lambda(x)}{c(x)}\left(e^{\mu(1-x)}+e^{\mu(x-1)}\right) .
$$


This implies for all $\mu \geq 0$ and all $x$ in $[0,1]$

$$
\left|G_{\mu \mu}(\mu, x)\right| \leq 2 \frac{\bar{\lambda}}{\underline{c}} e^{\mu} .
$$

Since,

$$
G(\mu, x)=\int_{0}^{\mu} \int_{0}^{r} G_{\mu \mu}(s, x) d s d r
$$

consequently, this yields for all $\mu \geq 0$ and all $x \in[0,1]$

$$
|G(\mu, x)| \leqslant \int_{0}^{\mu} \int_{0}^{r}\left|G_{\mu \mu}(s, x)\right| d s d r \leqslant \frac{\bar{\lambda}}{\underline{c}} e^{\mu} \mu^{2} .
$$

On one hand, it yields

$$
\begin{aligned}
F(p, \mu, x) & =\left(\sqrt{p}-e^{-\mu}\right)\left(2 \frac{\lambda(x)}{c(x)}+\mu\right) \\
& -e^{-\mu} \frac{\lambda(x)}{c(x)}\left(e^{\mu(1-x)}+e^{\mu(x-1)}\right) \\
& +e^{-\mu}\left(2 \frac{\lambda(x)}{c(x)}+\mu\right)-\frac{\lambda(x)}{c(x)}\left(p-e^{-2 \mu}\right) e^{\mu x} \\
& =\left(\sqrt{p}-e^{-\mu}\right)\left(2 \frac{\lambda(x)}{c(x)}+\mu\right) \\
& -\frac{\lambda(x)}{c(x)}\left(p-e^{-2 \mu}\right) e^{\mu x}+e^{-\mu} G(\mu, x)+e^{-\mu} \mu .
\end{aligned}
$$

Hence, with (58), this implies that

$$
\begin{gathered}
F(p, \mu, x) \geqslant e^{-\mu} \mu-\left|\sqrt{p}-e^{-\mu}\right|\left(2 \frac{\bar{\lambda}}{\underline{c}}+\mu\right) \\
\quad-\frac{\bar{\lambda}}{\underline{c}}\left|p-e^{-2 \mu}\right| e^{\mu}-\mu^{2} \frac{\bar{\lambda}}{\underline{c}} \\
\geqslant e^{-\mu} \mu-\left|\sqrt{p}-e^{-\mu}\right| \mu \\
-\frac{\bar{\lambda}}{\underline{c}}\left(2\left|\sqrt{p}-e^{-\mu}\right|+\left|p-e^{-2 \mu}\right| e^{\mu}+\mu^{2}\right) .
\end{gathered}
$$

On the other hand, 55 gives,

$$
\frac{\bar{\lambda}}{\underline{c}} \mu^{2}<\frac{e^{-\mu} \mu}{5}
$$

Moreover if $e^{-2 \mu} \geqslant p \geqslant C_{1}(\mu)$, it yields

$$
\left|e^{-\mu}-\sqrt{p}\right|=e^{-\mu}-\sqrt{p} \leqslant \frac{e^{-\mu}}{5} .
$$

Moreover, if $e^{-2 \mu} \geqslant p \geqslant C_{2}(\mu)$, it implies

$$
2 \frac{\bar{\lambda}}{\underline{c}}\left|e^{-\mu}-\sqrt{p}\right|=2 \frac{\bar{\lambda}}{\underline{c}}\left(e^{-\mu}-\sqrt{p}\right) \leqslant \frac{e^{-\mu} \mu}{5} .
$$

Finally, if $e^{-2 \mu} \geqslant p \geqslant C_{3}(\mu)$, it yields

$$
\frac{\bar{\lambda}}{\underline{c}}\left|e^{-2 \mu}-p\right| e^{\mu}=\frac{\bar{\lambda}}{\underline{c}}\left(e^{-2 \mu}-p\right) e^{\mu} \leqslant \frac{e^{-\mu} \mu}{5} .
$$

Consequently, this implies that (57) holds.

\section{E. Proof of Proposition 4}

First of all, note that with the definition of the function $V$ in (34), and since $p<1$, one gets

$$
\begin{aligned}
V(\phi, z) & \leqslant q|z|^{2}+\frac{1}{\underline{c}}\left\|\phi^{-}\right\|_{L^{2}(0,1)}^{2}+\frac{p e^{\mu}}{\underline{c}}\left\|\phi^{+}\right\|_{L^{2}(0,1)}^{2} \\
& \leqslant L_{1}\left(|z|+\left\|\phi^{-}\right\|_{L^{2}(0,1)}+\left\|\phi^{+}\right\|_{L^{2}(0,1)}\right)^{2}
\end{aligned}
$$

where $L_{1}=\max \left\{q, \frac{1}{\underline{c}}, \frac{p e^{\mu}}{\underline{c}}\right\}$. Also,

$$
\begin{aligned}
V(\phi, z) & \geqslant q|z|^{2}+\frac{e^{-\mu}}{\bar{c}}\left\|\phi^{-}\right\|_{L^{2}(0,1)}^{2}+\frac{p}{\bar{c}}\left\|\phi^{+}\right\|_{L^{2}(0,1)}^{2} \\
& \geqslant L_{2}\left(|z|+\left\|\phi^{-}\right\|_{L^{2}(0,1)}+\left\|\phi^{+}\right\|_{L^{2}(0,1)}\right)^{2},
\end{aligned}
$$

where $L_{2}=\min \left\{\frac{q}{3}, \frac{e^{-\mu}}{3 \bar{c}}, \frac{p}{3 \bar{c}}\right\}$. Moreover,

$$
\begin{aligned}
U(\xi, \phi, z) \leqslant|\xi| & +|n||z| \\
& +|m| \frac{\underline{c}+2 \bar{\lambda}}{\underline{c}^{2}}\left(\left\|\phi^{-}\right\|_{L^{2}(0,1)}+\left\|\phi^{+}\right\|_{L^{2}(0,1)}\right) \\
\leqslant & \leqslant L_{3}\|(\phi, z, \xi)\|_{\mathbb{X}} .
\end{aligned}
$$

where $L_{3}=\max \left\{1,|m| \frac{c+2 \bar{\lambda}}{\underline{c}^{2}},|n|\right\}$. Finally note that

$$
U(\xi, \phi, z)=\xi+Z(\phi, z)
$$

where

$$
Z(\phi, z)=m^{T} M(\phi)+n z \leqslant \sqrt{L_{3} V(\phi, z)} .
$$

where $L_{3}$ is a positive real number. Hence, it yields

$$
U(\xi, \phi, z)^{2} \geqslant|\xi|^{2}+Z(\phi, z)^{2}-2|\xi||Z(\phi, z)| .
$$

By completing the square this implies for all $0<\ell<1$

$$
\begin{aligned}
U(\xi, \phi, z)^{2} & \geqslant|\xi|^{2}(1-\ell)-\left(\frac{1}{\ell}-1\right)|Z(\phi, z)|^{2} \\
& \geqslant|\xi|^{2}(1-\ell)-\left(\frac{1}{\ell}-1\right) L_{3} V(\phi, z) .
\end{aligned}
$$

Finally, this yields setting $\ell$ sufficiently closed to 1 and a positive real number $L_{4}$ such that,

$$
\begin{aligned}
W(\xi, \phi, z) & \geqslant r|\xi|^{2}(1-\ell)+\left(1-r\left(\frac{1}{\ell}-1\right)\right) L_{3} V(\phi, z) \\
& \leqslant \frac{1}{L_{4}}\|(\phi, z, \xi)\|_{\mathbb{X}}^{2} .
\end{aligned}
$$

Hence, setting $L \geqslant \max \left\{L_{2}, L_{3}, L_{4}\right\}$, the result is obtained.

\section{F. Proof of Proposition 5}

Let $K_{p}$ be in $\left[\underline{K}_{p}, \bar{K}_{p}\right]$. First of all

$$
R(x)\left[\begin{array}{ll}
1 & 1 \\
1 & 1
\end{array}\right]=0 .
$$

Hence, we get the property

$$
\begin{aligned}
\dot{M}(t) & =\int_{0}^{1} \frac{I-R(x)}{c(x)} \phi_{t}(x, t) d x \\
= & \int_{0}^{1}(I-R(x))\left[\begin{array}{cc}
-1 & 0 \\
0 & 1
\end{array}\right] \phi_{x}(x, t) \\
& -\frac{\lambda(x)}{c(x)}\left[\begin{array}{cc}
1 & 1 \\
1 & 1
\end{array}\right] \phi(x, t) d x .
\end{aligned}
$$


Note that

$$
R_{x}(x)\left[\begin{array}{cc}
-1 & 0 \\
0 & 1
\end{array}\right]=\frac{\lambda(x)}{c(x)}\left[\begin{array}{ll}
1 & 1 \\
1 & 1
\end{array}\right] .
$$

Consequently, with an integration by parts, it yields

$$
\begin{aligned}
\dot{M}(t) & =(I-R(1))\left[\begin{array}{cc}
-1 & 0 \\
0 & 1
\end{array}\right] \phi(1, t)-\left[\begin{array}{cc}
-1 & 0 \\
0 & 1
\end{array}\right] \phi(0, t) \\
& =\left[\begin{array}{cc}
-1-\zeta & -\zeta \\
-\zeta & 1-\zeta
\end{array}\right] \phi(1, t)-\left[\begin{array}{cc}
-1 & 0 \\
0 & 1
\end{array}\right] \phi(0, t),
\end{aligned}
$$

where

$$
\zeta=\int_{0}^{1} \frac{\lambda(x)}{c(x)} d x
$$

Consequently,

$$
\begin{aligned}
\dot{U}(t) & =\phi^{-}(0, t)+\phi^{+}(0, t)-n(a+b) z(t)+n a \phi^{-}(1, t) \\
& +m_{1}\left(\phi^{-}(0, t)-(1+\zeta) \phi^{-}(1, t)-\zeta \phi^{+}(1, t)\right) \\
& +m_{2}\left(-\frac{\lambda}{2} \phi^{-}(1, t)+(1-\zeta) \phi^{+}(1, t)-\phi^{+}(0, t)\right)
\end{aligned}
$$

Employing the boundary conditions (32) and $\alpha_{p}$ defined in (33), it yields

$$
\begin{aligned}
& \dot{U}(t)=\phi^{-}(1, t)\left(a n-m_{1}(1+\zeta)-m_{2} \zeta-\frac{n(a+b)}{2}\right) \\
&+\phi^{+}(1, t)\left(m_{2}(1-\zeta)-m_{1} \zeta-\frac{n(a+b)}{2}\right) \\
&+\phi^{+}(0, t)\left(\alpha_{p}+1+m_{1} \alpha_{p}-m_{2}\right) \\
&+\xi(t) \frac{K_{i}}{1-K_{p}}\left(1+m_{1}\right) .
\end{aligned}
$$

Our aim is now to solve in $m_{1}, m_{2}$ and $n$ the system

$$
\left[\begin{array}{ccc}
-1-\zeta & -\zeta & \frac{a-b}{2} \\
-\zeta & 1-\zeta & -\frac{a+b}{2} \\
\alpha_{p} & -1 & 0
\end{array}\right]\left[\begin{array}{c}
m_{1} \\
m_{2} \\
n
\end{array}\right]=\left[\begin{array}{c}
0 \\
0 \\
-\alpha_{p}-1
\end{array}\right]
$$

It is possible with

$$
\begin{aligned}
n & =\frac{2\left(\alpha_{p}+1\right)}{a\left(1-\alpha_{p}+2 \zeta\left(1+\alpha_{p}\right)\right)+\left(\alpha_{p}+1\right) b}, \\
m_{2} & =\frac{+2 a \alpha_{p}}{a\left(1-\alpha_{p}+2 \zeta\left(1+\alpha_{p}\right)\right)+\left(\alpha_{p}+1\right) b}+1 \\
m_{1} & =\frac{2 a}{a\left(1-\alpha_{p}+2 \zeta\left(1+\alpha_{p}\right)\right)+\left(\alpha_{p}+1\right) b}-1 .
\end{aligned}
$$

These values are defined for almost all $\alpha_{p}$ hence for almost all $K_{p}$ in $\left[\underline{K}_{p}, \bar{K}_{p}\right]$. In that case, it yields

$$
\dot{U}(t)=\xi(t) \frac{K_{i}}{1-K_{p}}\left(1+m_{1}\right)
$$

So we select $K_{i}$ such that $\frac{K_{i}}{1-K_{p}}\left(1+m_{1}\right)<0$.

Hence, we get

$$
\begin{aligned}
\frac{2 U(t) \dot{U}(t)}{\left|K_{i}\right|} & \leqslant-\xi(t)^{2}\left|\frac{1+m_{1}}{1-K_{p}}\right|+\xi(t) m^{\top} M(t)+\xi(t) n z \\
& \leqslant-c_{1} \xi(t)^{2}+c_{2} V(t),
\end{aligned}
$$

where $c_{1}$ and $c_{2}$ are obtained by applying the Cauchy Schwartz inequality and by completing the square. Finally, this with 35 yield

$$
\dot{W}(t) \leqslant\left(c_{2} r\left|K_{i}\right|-\omega_{1}\right) V(t)+\left(\delta-r c_{1}\right)\left|K_{i}\right| \xi(t)^{2} .
$$

So, we select

$$
0<\left|K_{i}\right|<\frac{\omega_{1} c_{1}}{\delta c_{2}} .
$$

Hence, we can choose $r$ such that

$$
\frac{\delta}{c_{1}}<r<\frac{\omega_{1}}{c_{2}\left|K_{i}\right|} \text {. }
$$

This implies the existence of a positive real number $\omega_{2}$ such that equation 39 holds.

\section{G. Proof of Proposition 6}

Since inequality (39) is satisfied by assumption and exhibiting all the terms in which $\psi$ shows up in the time derivative of the Lyapunov function, it yields along the solution of the system :

$$
\begin{aligned}
& \dot{W}(t) \leqslant-\omega_{2} W(t) \\
& \quad+2 \int_{0}^{1} \frac{\psi(x)}{c(x)^{2}} \phi(x, t)^{\top}\left[\begin{array}{cc}
e^{-\mu x} & 0 \\
0 & p e^{\mu x}
\end{array}\right]\left[\begin{array}{ll}
-1 & 1 \\
-1 & 1
\end{array}\right] \phi(x, t) d x \\
& \quad+2 r U(t) m^{\top} M\left(\frac{\psi(\cdot)}{c(\cdot)}\left[\begin{array}{cc}
-1 & 1 \\
-1 & 1
\end{array}\right] \phi(\cdot, t)\right),
\end{aligned}
$$

where $M$ is the bounded linear operator defined in (37).

Note that

$$
\begin{array}{r}
2 \int_{0}^{1} \frac{\psi(x)}{c(x)^{2}} \phi(x, t)^{\top}\left[\begin{array}{cc}
e^{-\mu x} & 0 \\
0 & p e^{\mu x}
\end{array}\right]\left[\begin{array}{cc}
-1 & 1 \\
-1 & 1
\end{array}\right] \phi(x, t) d x \\
\leqslant \mathfrak{r}_{1} \bar{\psi} V(t) \leqslant \mathfrak{r}_{1} \bar{\psi} W(t),
\end{array}
$$

with $\mathfrak{r}_{1}$ is a positive real number (depending on $\underline{c}, p$ and $\mu$ ) such that for all $x$ in $[0,1]$

$$
\frac{1}{c(x)}\left[\begin{array}{cc}
-2 e^{-\mu x} & e^{-\mu x}-p e^{\mu x} \\
e^{-\mu x}-p e^{\mu x} & 2 p e^{\mu x}
\end{array}\right] \leqslant \mathfrak{r}_{1}\left[\begin{array}{cc}
e^{-\mu x} & 0 \\
0 & p e^{\mu x}
\end{array}\right] .
$$

Also, note that since $r U(t)^{2} \leqslant W(t)$, it yields

$$
\begin{aligned}
& 2 r U(t) m^{\top} M\left(\frac{\psi(\cdot)}{c(\cdot)}\left[\begin{array}{ll}
-1 & 1 \\
-1 & 1
\end{array}\right] \phi(\cdot)\right) \\
& \quad \leqslant \mathfrak{r}_{2} \bar{\psi} \sqrt{W(t)}\|\phi(\cdot, t)\|_{\left(L^{1}(0,1)\right)^{2}},
\end{aligned}
$$

where

$$
\mathfrak{r}_{2}=2 \sqrt{r}|m|\|M\|_{\left.\left(L^{1}(0,1)\right)^{2} ; \mathbb{R}^{2}\right)} \frac{1}{\underline{c}}\left\|\left[\begin{array}{ll}
-1 & 1 \\
-1 & 1
\end{array}\right]\right\| .
$$

With Hölder inequality, and Proposition 4 it yields a positive real number $\mathfrak{r}_{3}$ such that

$$
\mathfrak{r}_{2}\|\phi(\cdot, t)\|_{\left(L^{1}(0,1)\right)^{2}} \leqslant \mathfrak{r}_{3} \sqrt{W(t)}
$$

In conclusion,

$$
\dot{W}(t) \leqslant\left(-\omega_{2}+\left(\mathfrak{r}_{1}+\mathfrak{r}_{3}\right) \bar{\psi}\right) W(t)
$$

Hence, with

$$
\bar{\psi}=\frac{\omega_{2}}{2\left(\mathfrak{r}_{1}+\mathfrak{r}_{3}\right)}
$$

equation 40 holds. 


\section{H. Proof of Proposition 7}

Consider the system of equations (11)-(15) with $\lambda(x)=$ $\psi(x)=0$ and $c(x)=c$ constant and $b \geqslant 0$. Employing the Laplace transform the following equalities for the dynamics may be obtained

$$
\begin{gathered}
s \xi(s)=\phi^{-}(0, s)+\phi^{+}(0, s) \\
z(s)=\frac{a}{s+a+b} \phi^{-}(1, s) \\
\phi^{-}(1, s)=\phi^{-}(0, s) e^{-c s}, \quad \phi^{+}(0, s)=\phi^{-}(1, s) e^{-c s}
\end{gathered}
$$

At $x=0$ the boundary condition (14) imposes:

$$
\begin{aligned}
& s\left(1-K_{p}\right) \phi^{-}(0, s)= \\
& \quad s \alpha_{p}\left(1-K_{p}\right) \phi^{+}(0, s)+K_{i}\left(\phi^{-}(0, s)+\phi^{+}(0, s)\right),
\end{aligned}
$$

which gives

$$
\phi^{-}(0, s)=\frac{s \alpha_{p}\left(1-K_{p}\right)+K_{i}}{s\left(1-K_{p}\right)-K_{i}} \phi^{+}(0, s)
$$

while at $x=1$, conditions $(15)$ becomes:

$$
\begin{gathered}
\phi^{+}(1, s)=-\phi^{-}(1, s)+\frac{2 a}{s+a+b} \phi^{-}(1, s) \\
\phi^{+}(1, s)=\frac{a-s-b}{s+a+b} \phi^{-}(1, s) \\
=\frac{(a-s-b)}{(s+a+b)} \frac{\left(s \alpha_{p}\left(1-K_{p}\right)+K_{i}\right)}{\left(s\left(1-K_{p}\right)-K_{i}\right)} e^{-2 c s} \phi^{+}(1, s)
\end{gathered}
$$

Thus, we obtain a characteristic equation of the form

$(s+a+b)\left(s-\frac{K_{i}}{\left(1-K_{p}\right)}\right)+(s+b-a)\left(s \alpha_{p}+\frac{K_{i}}{\left(1-K_{p}\right)}\right) e^{-2 c s}=0$

Set $K=-\frac{K_{i}}{1-K_{p}}$ to get the following characteristic equation

$$
\left.(s+a+b)(s+K)+(s+b-a)\left(s \alpha_{p}-K\right)\right) e^{-2 c s}=0
$$

We follow the Walton and Marshall procedure as is it used in [2] and described in [18]

First step : the roots of (66) are examined with $c=0$

In this case, one looks for the roots of

$$
\left(1+\alpha_{p}\right) s^{2}+\left(a+b+\alpha_{p}(b-a)\right) s+2 a K=0
$$

which are

$$
\begin{aligned}
s_{1} & =-\frac{a\left(1-\alpha_{p}\right)+b\left(1+\alpha_{p}\right)}{2\left(1+\alpha_{p}\right)} \\
& +\frac{\sqrt{\left(a\left(1-\alpha_{p}\right)+b\left(1+\alpha_{p}\right)\right)^{2}-8 a K\left(1+\alpha_{p}\right)}}{2\left(1+\alpha_{p}\right)} \\
s_{1} & =-\frac{a\left(1-\alpha_{p}\right)+b\left(1+\alpha_{p}\right)}{2\left(1+\alpha_{p}\right)} \\
& -\frac{\sqrt{\left(a\left(1-\alpha_{p}\right)+b\left(1+\alpha_{p}\right)\right)^{2}-8 a K\left(1+\alpha_{p}\right)}}{2\left(1+\alpha_{p}\right)}
\end{aligned}
$$

Provided that

$$
\operatorname{sgn}\left(K_{i}\right)=\operatorname{sgn}\left(K_{p}-1\right), \quad K_{i} \neq 0
$$

the system poles have strictly negative real part for $c=0$.
Second step : We compute the polynomial in $w^{2}$ :

$$
W\left(w^{2}\right) \triangleq d(j w) d(-j w)-n(j w) n(-j w)
$$

with

$$
d(x)=(x+a+b)(x+K) \text { and }
$$$$
n(x)=(x+b-a)\left(x \alpha_{p}-K\right) \text { then, }
$$

$$
\begin{aligned}
W(X)= & \left(X+(a+b)^{2}\right)\left(X+K^{2}\right) \\
& -\left(X+(b-a)^{2}\right)\left(\alpha_{p}^{2} X+K^{2}\right) \\
= & \left(1-\alpha_{p}^{2}\right) X^{2}+\left((a+b)^{2}-\alpha_{p}^{2}(b-a)^{2}\right) X+4 a b K^{2}
\end{aligned}
$$

where $X=w^{2}$. Notes that $\left.K_{p} \in\right]-\infty ; \frac{1-\alpha_{0}}{2}\left[\Rightarrow\left|\alpha_{p}\right|<1\right.$. It implies that the sign of the polynomial $W$ is positive for large $X$. We can deduce that the poles of the system have strictly negative real parts for sufficiently small value of $c$.

Third step : Computing the roots of $W(X)$

We obtain

$$
\begin{aligned}
& X_{1}=\frac{-\left(\left(a^{2}+b^{2}\right)\left(1-\alpha_{p}^{2}\right)+2 a b\left(1+\alpha_{p}^{2}\right)\right)}{2\left(1-\alpha_{p}^{2}\right)}+ \\
& \frac{\sqrt{\left(\left(a^{2}+b^{2}\right)\left(1-\alpha_{p}^{2}\right)+2 a b\left(1+\alpha_{p}^{2}\right)\right)^{2}-16 a b K^{2}\left(1-\alpha_{p}^{2}\right)}}{2\left(1-\alpha_{p}^{2}\right)} \\
& X_{2}=\frac{-\left(\left(a^{2}+b^{2}\right)\left(1-\alpha_{p}^{2}\right)+2 a b\left(1+\alpha_{p}^{2}\right)\right)}{2\left(1-\alpha_{p}^{2}\right)}- \\
& \frac{\sqrt{\left(\left(a^{2}+b^{2}\right)\left(1-\alpha_{p}^{2}\right)+2 a b\left(1+\alpha_{p}^{2}\right)\right)^{2}-16 a b K^{2}\left(1-\alpha_{p}^{2}\right)}}{2\left(1-\alpha_{p}^{2}\right)}
\end{aligned}
$$

which have strictly negative real parts since

$\left(\left(a^{2}+b^{2}\right)\left(1-\alpha_{p}^{2}\right)+2 a b\left(1-\alpha_{p}^{2}\right)\right)>0, a b K^{2}\left(1-\alpha_{p}^{2}\right)>0$

for all positive parameters.

After these three steps, we can conclude that for every negative value of $K_{i}$, the poles of the system governed by (11)-(15) in the special case where $\lambda(x)=\psi(x)=0$ and $c(x)=c$ are stable whatever the length $L$ or the velocity $c$.

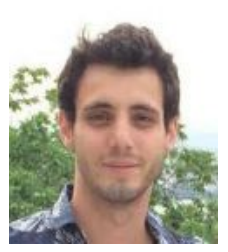

Alexandre Terrand-Jeanne graduated in electrical engineering from ENS Cachan, France, in 2013. After one year in the robotic laboratory "Centro E.Piaggio" in Pisa, Italy, he is currently a doctoral student in LAGEP, university of Lyon 1. His PhD topic concerns the stability analysis and control laws design for systems involving hyperbolic partial differential equations coupled with nonlinear ordinary differential equations. This work is under the supervision of V. Dos Santos Martins, V. Andrieu and M. Tayakout-Fayolle. 


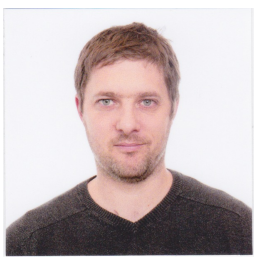

Vincent Andrieu graduated in applied mathematics from INSA de Rouen, France, in 2001. After working in ONERA (French aerospace research company), he obtained a $\mathrm{PhD}$ degree from Ecole des Mines de Paris in 2005. In 2006, he had a research appointment at the Control and Power Group, Dept. EEE, Imperial College London. In 2008, he joined the CNRS-LAAS lab in Toulouse, France, as a CNRS-chargé de recherche. Since 2010, he has been working in LAGEP-CNRS, Université de Lyon 1, France. In 2014, he joined the functional analysis group from Bergische Universität Wuppertal in Germany, for two sabbatical years. His main research interests are in the feedback stabilization of controlled dynamical nonlinear systems and state estimation problems. He is also interested in practical application of these theoretical problems, and especially in the field of aeronautics and chemical engineering.

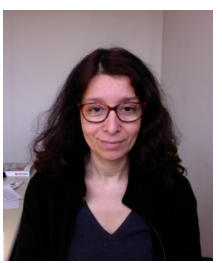

Mélaz Tayakout-Fayolle is a Full Professor with the LAGEP (Laboratoire dAutomatique et de GEnie des Procédés) of University of Lyon 1 . She received her BSc in Physics and Chemistry and MSc in Industrial Chemistry from the University of Marseille. Her $\mathrm{Ph} . \mathrm{D}$. degree in Chemical Engineering was obtained in 1991 from the University of Lyon. She starded as an Associated Professor at LAGEP (Laboratoire dAutomatique et Génie des Procédés) of the University of Lyon. Prior to joining IRCELYON as Full Professor, she worked three years as Research Engineer for IFPEN Compagny. Since 1991, she has taught courses in chemical engineering, thermodynamics, mass transfer, dynamical modelling. Her research areas of interest include modelling of triphasic reactors: mass transfer, chemical kinetics of the complex matrix and thermodynamics, and design concepts in reactors. The complex matrix concerns the heaviest fractions of petroleumin in hydroconversion and hydrocracking processes. Since 2014, she is a consultant for the Total Company.

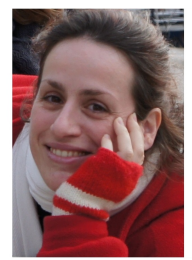

Valérie Dos Santos Martins graduated in Mathematics from the University of Orléans, France in 2001. She received the Ph.D degree in 2004 in Applied Mathematics from the University of Orléans. After one year in the laboratory of Mathematics MAPMO in Orléans as ATER, she was post-doct in the laboratory CESAME/INMA of the University Catholic of Louvain, Belgium. Currently, she is professor assistant in the laboratory LAGEP, University of Lyon 1. Her current research interests include nonlinear control theory, perturbations theory of operators and semigroup, spectral theory and control of nonlinear partial differential equations. 